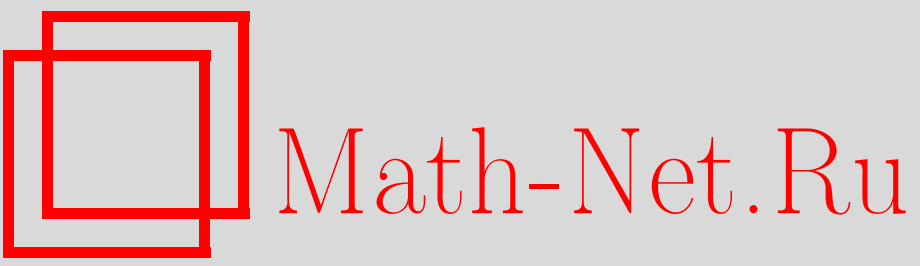

А. В. Тищенко, Моноид полугрупповых многообразий относительно сплетения, УМH, 1996, том 51, выпуск 2, 177-178

DOI: https://doi.org/10.4213/rm964

Использование Общероссийского математического портала Math-Net.Ru подразумевает, что вы прочитали и согласны с пользовательским соглашением

http://www.mathnet.ru/rus/agreement

Параметры загрузки:

IP: 54.209 .52 .79

26 апреля 2023 г., 10:40:26 


\title{
МОНОИД ПОЛУГРУППОВЫХ МНОГООБРАЗИЙ ОТНОСИТЕЛЬНО СПЛЕТЕНИЯ
}

\author{
А. В. Тишенко
}

Операция сплетения полугрупповых многообразий определена в [1], [2]. Мы называем ее моноидным сплетением для того, чтобы отличить ее от других возможных операций на многообразиях, введенных с помощью сплетения полугрупп. Заметим, что на периодических групповых многообразиях моноидное сплетение совпадает с произведением групповых многообразий. Интерес к изучению этой операции вызван, с одной стороны, тем, что эта операция показала свою плодотворность при изучении многообразий групп [3], а с другой стороны, тем, что она возникла в теории декомпозиции конечных полугрупп, а также при изучении формальных языков и конечных автоматов [4], [5]. При этом изучение сплетения полугрупповых многообразий содержит очевидные перспективы применения получаемых результатов к псевдомногообразиям конечных полугрупп в силу близости понятий многообразия и псевдомногообразия (см., например, [6]).

Сплетением $(S w R \mid A)$ полугрупп $S$ и $R$ при помощи правого $R$-полигона $A$ называется полугруппа, определенная на множестве $S^{A} \times R$ посредством умножения, заданного формулой $(f, p)(g, q)=\left(f^{p} g, p q\right)$, где $\left(f^{p} g\right)(a)=f(a) g(a p)$ для всех $f, g \in S^{A}, a \in A, p, q \in R$ (см. [7], [8]). Сплетение назовем расширенным стандартным, если $A=R^{1}$, где $R^{1}$ - наименьший моноид, содержащий полугруппу $R$. Расширенное стандартное сплетение обозначим через $S w R$.

Моноидным сплетением полугрупповых многообразий $\boldsymbol{U}$ и $\boldsymbol{V}$ назовем полугрупповое многообразие $\boldsymbol{U} w \boldsymbol{V}$, порожденное всевозможными расширенными стандартными сплетениями $S w R$, в которых $S \in \boldsymbol{U}$ и $R \in \boldsymbol{V}$. Очевидно, что многообразие $\boldsymbol{S}$ всех полугрупп является нулем, а многообразие $\boldsymbol{T}$ всех одноэлементных (тривиальных) полугрупп является единицей относительно моноидного сплетения многообразий. Прежде всего, укажем на два известных результата об операции моноидного сплетения.

Теорема 1 [1]. Моноидное сплетение полугрупповых многообразий ассоциативно.

Теорема 2 [9], [6]. Многообразие полугрупп является идемпотентом относительно моноидного сплетения многообразий тогда и только тогда, когда оно есть либо многообразие $\boldsymbol{S}$ всех полугрупп, либо совпадает с одним из многообразий $\boldsymbol{L}_{j, m}$, задаваемым одним тождеством

$$
x_{1} \ldots x_{j} y_{1} \ldots y_{m-j}=x_{1} \ldots x_{j} z_{1} \ldots z_{m-j+1} \quad(m \geqslant 1,0 \leqslant j \leqslant m) .
$$

Отметим, что при $j=0$ многообразие $\boldsymbol{L}_{0, m}$ есть многообразие $\boldsymbol{N}_{m}$ нильпотентных полугрупп индекса $m$. В частности, $\boldsymbol{L}_{0,1}$ есть многообразие $\boldsymbol{T}$ тривиальных полугрупп. Полугруппа называется нильпотентной слева индекса $m$, если произведение любых ее $m$ элементов есть левый нуль полугруппы. При $j=m$ многообразие $\boldsymbol{L}_{m, m}$ есть класс всех нильпотентных слева полугрупп индекса $m$.

Пусть $M V$ - моноид всех полугрупповых многообразий относительно моноидного сплетения. Все полугрупповые многообразия разобьем на пять классов: $T$ - одноэлементное множество, состоящее только из многообразия $\boldsymbol{T}$ тривиальных полугрупп; $L N$ - множество всех нетривиальных периодических многообразий, состоящих из нильпотентных слева полугрупп; $G$ - множество всех периодических групповых многообразий; $O C$ - множество всех надкоммутативных многообразий (т.е. содержащих многообразие коммутативных полугрупп); $F$ - множество всех периодических многообразий, содержащих полугруппу, которая не является ни группой, ни нильпотентной слева полугруппой.

Теорема 3. Моноид $M V$ полугрупповых многообразий можно представить как пятиэлементную полурешетку своих непересекающихся подполугрупп $T, L N, G, F, O C$, в которой $T$ - наибольший әлемент, OC - наименьиий әлемент, $F \leqslant L N u F \leqslant G$, а $L N$ и $G$ не сравнимы. При этом $O C$ - полугруппа с нулевым умножением, $G$ свободная полугруппа континуального ранга, $F$ - полугруппа без идемпотентов, не 
удовлетворяющая ни правому, ни левому закону сокращения, а компонента LN разложима в счетную полурешетку конечных нильпотентных полугрупп $T\left(\boldsymbol{L}_{j, m}\right)$, где $m \geqslant 1,0 \leqslant j \leqslant m, m+j \geqslant 2$.

Что можно сказать о строении компонент полурешеточного разложения $M V$, полученного в теореме 3 ? Нилпотентные компоненты $O C$ и $T\left(\boldsymbol{L}_{j, m}\right)$, очевидно, далее полурешеточно неразложимы. Компонента $T$ состоит из одного элемента, а $G$ имеет понятное полугрупповое строение. Для компоненты $G$ нет описания множества свободных образующих, т.е. всех неразложимых в сплетение многообразий. С другой стороны, моноидное сплетение периодических групповых многообразий совпадает с их произведением, и поэтому достаточно большое число примеров неразложимых групповых многообразий имеется в книге [3]. Полное описание неразложимых групповых многообразий пока отсутствует.

Наиболее неясным остается сейчас строение компоненты $F$. О структуре полугруппы $F$ известна пока лишь информация, указанная в теореме 3 . Более точное изучение компоненты $F$ пока представляет известные трудности, так как в случае полугрупп без кручения дальнейшие полурешеточные разложения могут не привести к компонентам, устроенным более просто, чем исходная полугруппа $F$.

Дополнительную информацию о строении моноида $M V$ дают следующие два результата. Первый из них показьвает, что индексы нильпотентности конечных нилппотентных полугрупп $T\left(\boldsymbol{L}_{j, m}\right)$, фигурирующих в теореме 3 , не ограничены в совокупности. Второй результат дает полное описание условий на многообразия $\boldsymbol{U}$ и $\boldsymbol{V}$, при которых их сплетение равно нулю моноида $M V$, т.е. совпадает с $\boldsymbol{S}$.

Теорема 4. Для любого $m \geqslant 1$ индекс нильпотентности класса кручения $T\left(\boldsymbol{N}_{m}\right)$ равен $\left[\frac{m+1}{2}\right]$.

Теорема 5. Моноидное сплетение $\boldsymbol{U} \boldsymbol{w} \boldsymbol{V}$ двух полугрупповых многообразий равно $\boldsymbol{S}$ тогда и только тогда, когда $\boldsymbol{V}$ надкоммутативно, а $\boldsymbol{U}$ содержит либо неодноэлементную полурешетку, либо нетривиальную группу, либо счетнопорожденную коммутативную ненильпотентную полугруппу $Q$ с тождеством $x^{2}=0$.

В заключение отметим, что теорема 4 была анонсирована в [10], а теорема 3 улучшает результат о разложении моноида $M V$ в трехэлементную полурешетку, анонсированный в [10].

\section{СПИСОК ЛИТЕРАТУРЫ}

[1] Tilson B. // J. Pure and Appl. Algebra. 1987. V. 48. P. 83-198. [2] Тищенко А. В. // Междунар. конф. по алгебре памяти А.И. Ширшова (Барнаул, август 1991). Тезисы докл. по логике и универс. алгебрам, прикл. алгебре. Новосибирск, 1991. С. 144. [3] Нейман Х. Многообразия групп. М.: Мир, 1969. [4] Eilenberg S. Automata, Languages and Machines. V. B. New York: Acad. Press, 1976. [5] Лаллеман ЖK. Полугруппы и комбинаторные приложения. М.: Мир, 1985. [6] Almeida J. // Semigroup Forum. 1990. V. 40. P. 315-323. [7] Skornjakov L. A. // Semigroup Forum. 1979. V. 18. P. 83-86. [8] Тищенко А. В. // Сиб. матем. журн. 1986. Т. 27. №6. C. 183-195. [9] Koselev Yu. G. // Semigroup Forum. 1976. V. 12. P. 95-107. [10] Тищенко А. В. // Третья междунар. конф. по алгебре памяти М. И. Каргаполова (Красноярск, 23-28 августа 1993). Тезисы докл. Красноярск, 1993. С. 331.

Московский институт-интернат для инвалидов с нарушением опорно-двигательной системы
Принято редколлегией 24.11.1995 